\title{
Larix decidua (Europäische Lärche) - Baum des Jahres 2012
}

\author{
Veit Martin Dörken
}

\begin{abstract}
The European larch is tree of the year 2012. Morphology, biology, ecology and use are outlined. In addition the important forestry larchs (L. kaempferi and L. x eurolepis) are mentioned.
\end{abstract}

\section{Zusammenfassung}

Die Europäische Lärche (Larix decidua) ist Baum des Jahres 2012. Morphologie, Biologie, Ökologie und Verwendung werden erläutert. Ergänzend werden weitere forstwirtschaftlich wichtige Lärchen (L. kaempferi und L. x eurolepis) vorgestellt.

\section{Einleitung}

Die Europäische Lärche (Larix decidua = L. europaea) ist der einzige heimische Nadelbaum, der jährlich am Ende der Vegetationsperiode seine Nadeln abwirft. Hierauf bezieht sich auch der Art-Beiname decidua (= abfallend). Alle übrigen heimischen Koniferen wie Weiß-Tanne (Abies alba), Gewöhnliche Fichte (Picea abies), Wald- und Berg-Kiefer (Pinus sylvestris und P. mugo) sowie Gewöhnlicher Wacholder und Sadebaum (Juniperus communis und J. sabina) sind immergrün. Durch ihre prächtige, goldgelbe Herbstfärbung ist die Lärche ein auffälliges Nadelgehölz, das häufig als Forstbaum gepflanzt wird. Die Europäische Lärche wurde vom Kuratorium Baum des Jahres zum Jahresbaum 2012 gewählt.

\section{Systematik}

Die Gattungsname Larix wurde von der römischen Bezeichnung für die Lärche übernommen. Larix (Pinaceae) umfasst etwa 12 Arten, in Europa ist nur L. decidua heimisch (MABBERLEY 2008). Die Angaben über die tatsächliche Artenzahl schwanken erheblich, da besonders die ostasiatischen Arten morphologisch recht variabel sind. Die Gattung Larix wird in zwei Sektionen unterteilt, nämlich Larix (mit kurzen Deckschuppen) und Multiseralis (mit langen Deckschuppen). Die Europäische Lärche gehört zur Sektion Larix. In der klassischen Systematik der Kieferngewächse wurden die Lärchen allein aufgrund der ähnlichen Diffe- renzierung in Lang- und Kurztriebe zusammen mit den immergrünen Zedern (Cedrus spp.) und der winterkahlen Goldlärche (Pseudolarix amabilis) zur Unterfamilie der Laricoideae (den Lärchenartigen) zusammengefasst. Neuere morphologische und molekularphylogenetische Untersuchungen belegen jedoch eine engere Verwandtschaft von Lärchen mit Douglasien (Pseudotsuga spp., WANG et al. 2000).

Larix decidua kann in drei Varietäten unterteilt werden. Diese unterscheiden sich hauptsächlich durch die Größe ihrer Zapfen und die Färbung der Triebe.

Die Varietät decidua (Alpen-Lärche) hat $2-4 \mathrm{~cm}$ lange und $2-3 \mathrm{~cm}$ breite Zapfen. Der obere Rand ihrer Schuppen ist ganzrandig oder etwas eingebuchtet bis gewellt.

Bei der Varietät carpatica (Karpaten-Lärche) sind die Zapfen mit 3,5-6 cm Länge und $2-4 \mathrm{~cm}$ Breite größer. Der obere Rand der Schuppen ist ganzrandig und deutlich abgerundet. Nur 1-1,5 cm lange sowie breite Zapfen zeigt die Varietät polonica (Polnische Lärche). Die Zapfenschuppen sind konvex und fast kreisrund, ihr oberer Rand ist ganzrandig und abgerundet.

\section{Verbreitung und Vorkommen}

Die Europäische Lärche (Larix decidua) ist ein eurasischer Gebirgsbaum, der in den Alpen und den Karpaten heimisch ist. Sie kommt in hochmontanen bis subalpinen Nadelwaldstufen von 1600-2000 m ü.NN und teilweise auch darü- 
ber in Bergnadelwäldern oder an Rutschhängen vor. Als Pioniergehölz gedeiht die Art auch auf ärmsten Substraten wie z.B. Moränenschutt. Die Lärche bildet meist keine Reinbestände, sondern tritt in Mischbeständen auf. In Höhen unterhalb von $1800 \mathrm{~m}$ ist sie häufig mit Picea abies (Rot-Fichte), oberhalb oft mit Pinus cembra (Zirbel-Kiefer) vergesellschaftet.

In Deutschland tritt wild nur die Varietät decidua auf, welche ursprünglich ausschließlich im südlichen Bayern heimisch war. In den übrigen Teilen Deutschlands ist sie allerdings heute bis ins Flachland zu einem bedeutenden Forstgehölz geworden, verwildert auch dort regelmäßig und ist in einigen Flachlandregionen bereits als eingebürgert anzusehen. Hier findet man Naturverjüngungen besonders an bodensauren Standorten. Zum optimalen Gedeihen benötigt die Europäische Lärche offene, voll besonnte Standorte und verträgt schattige Lagen nur schlecht. Mit zunehmendem Schattendruck wächst die Art schief zum Licht hin, wie dies auch bei anderen heimischen, lichthungrigen Gehölzen zu beobachten ist (z. B. bei der Kornelkirsche). Die Europäische Lärche ist sehr frosthart. Sie verträgt aber weder Hitze noch Boden- und Luftverschmutzungen. Auch schwere, undurchlässige Tonböden sind ungeeignet. Besonders wüchsig ist sie auf locker-steinigen und tiefgründigen Lehmböden mit einer schwach sauren bis alkalischen Bodenreaktion. Empfindlich reagiert die windbedürftige Art auch gegenüber warmer Stauluft (Kiermeier 1993).

\section{Morphologie}

\subsection{Wuchs, Stamm und Zweige}

Die Europäische Lärche wird unter geeigneten Bedingungen bis $40 \mathrm{~m}$ hoch. Sie ist ein Herzwurzler mit einem hohen Anteil an Faserwurzeln im oberen Bodenhorizont. Ihre Krone ist schlank kegelförmig. Das Sprosssystem ist wie bei allen Lärchen in Lang- und Kurztriebe differenziert. Junge Triebe sind zunächst gelblich bis

Abb. 1 (oben): Lärchenwald im Martelltal/Südtirol.

Abb. 2 (unten): Lärchenzweig mit benadelten Kurztrieben.
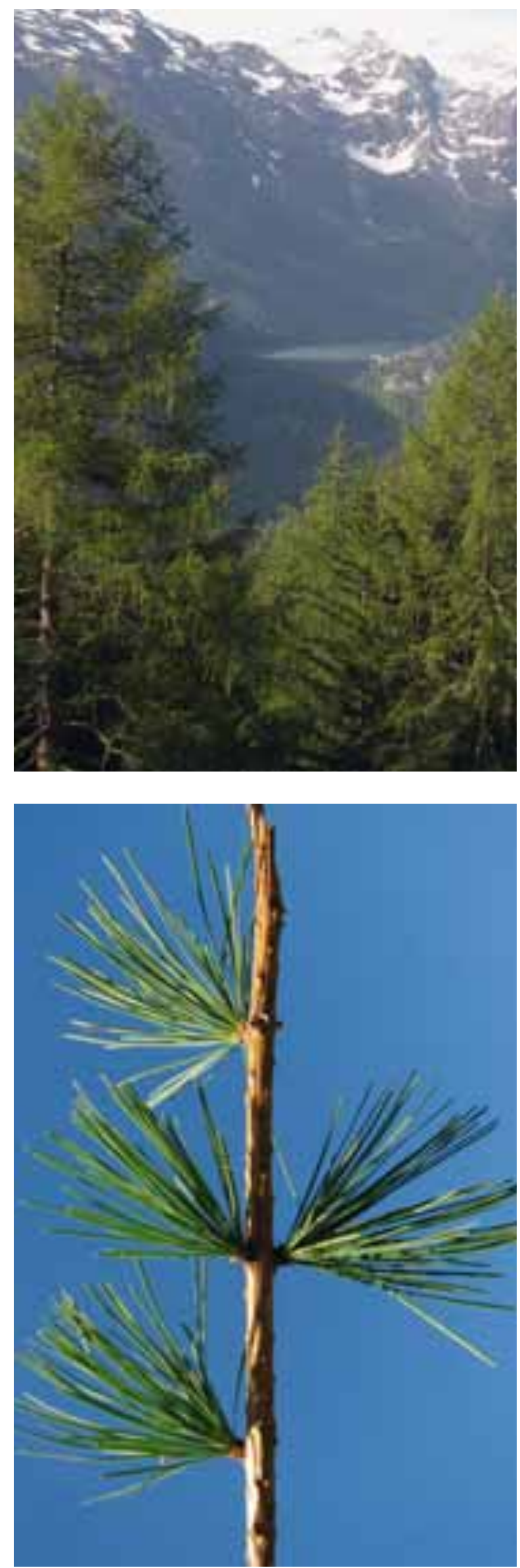

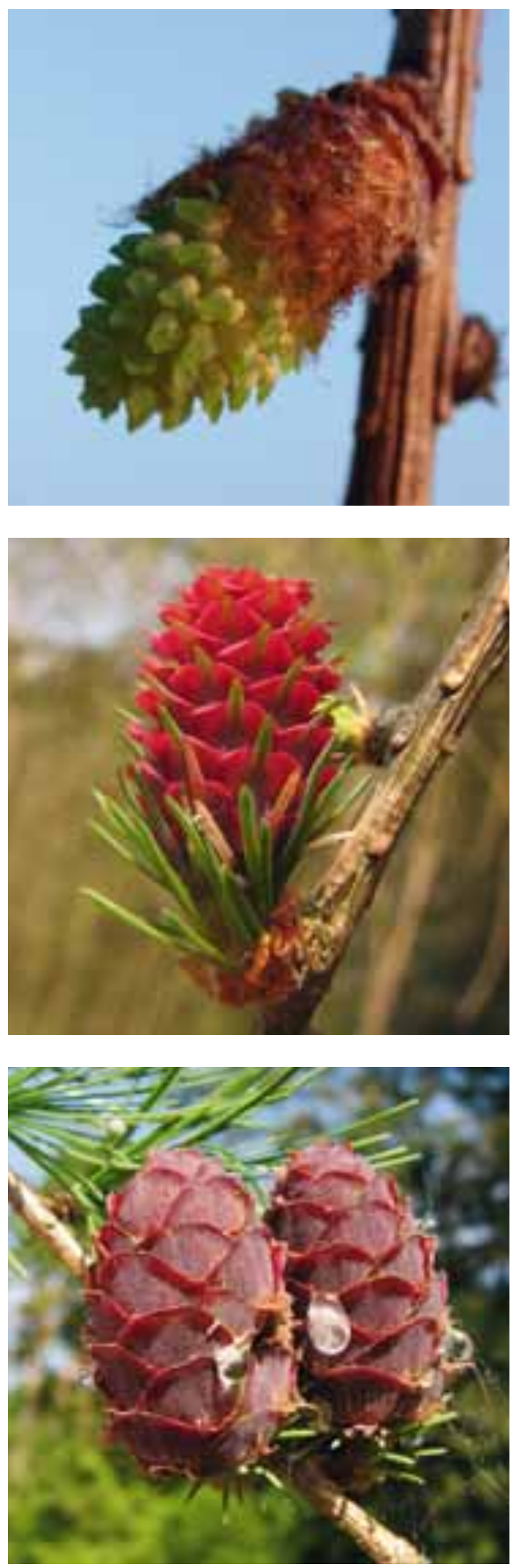

beigefarben oder weißlich und stets kahl. Die Borke der Stämme ist graubraun, im Alter stark längsrissig und blättert in kleinen Platten ab. Bei allen Lärchen-Arten ist ein regelmäßiges Absterben der Seitenäste von der Stammbasis in Richtung Gipfel zu beobachten, was durch Lichtmangel begründet sein dürfte. Unter Lärchen liegen daher oft zahlreiche abgeworfene Triebe. Der Abwurf der Triebe erfolgt dabei ohne ein spezielles Trennungsgewebe. Da bei Larix der Zapfen immer aus der einzigen Endknospe eines Kurztriebes hervorgeht und dabei der Vegetationspunkt aufgebraucht wird, ist ein zapfenbesetzter Kurztrieb nicht mehr in der Lage, weiterzuwachsen. Im Gegensatz zu den übrigen Kieferngewächsen fallen bei Lärchen die Zapfen aber weder als Ganzes ab, noch zerfallen sie, sondern bleiben über viele Jahre am Baum. Deshalb muss für eine ausreichende mechanische Stabilisierung zapfenbesetzter Triebe und kommender Sprossgenerationen viel Biomasse in diese Triebe investiert werden. Um dies zu vermeiden, gehen bei Larix solche Triebe nach einiger Zeit verloren. Daher ist der Verlust zapfenbesetzter Triebe ein wichtiger Prozess im Zuge der Selbstreinigung zum Erhalt einer transparenten Krone (Dörken 2012).

\subsection{Nadeln}

Eine Besonderheit der Lärchennadeln ist, dass sie im Herbst abgeworfen werden. Weltweit sind winterkahle Nadelbäume heutzutage sehr selten. Neben den Larix-Arten kommt der Laubabwurf noch innerhalb der Kieferngewächse bei der Goldlärche (Pseudolarix amabilis) und innerhalb der Zypressengewächse beim Urweltmammutbaum (Metasequoia glyptostroboides), den Sumpfzypressen (Taxodium), der Wasserfichte (Glyptostrobus pensilis) und beim Ginkgo (Ginkgo biloba, Ginkgoaceae) vor.

Da die Nadeln nur eine Vegetationsperiode lang ausdauern müssen, sind sie vergleichsweise

Abb. 3 (oben): Junger Pollenzapfen.

Abb. 4 (Mitte): Junger weiblicher Zapfen.

Abb. 5 (unten): Ältere weibliche Zapfen. 
weich. Sie sind an Kurz- und Langtrieben gleich groß und weisen auf der Unterseite zwei deutliche, weiße Spaltöffnungsreihen auf. An den Langtrieben stehen sie gleichmäßig verteilt, in einigen Blattachseln entwickeln sich Kurztriebe, an denen die Nadeln zu 30-40 dicht büschelig gedrängt stehen. Der Laubabwurf erfolgt erst im November. Die Lärche ist damit zusammen mit den heimischen Birken-Arten (Betula pendula und B. pubescens) die am spätesten ausfärbende heimische Baumart. Die abgeworfenen Nadeln zersetzen sich aufgrund eines sehr ungünstigen Kohlenstoff/Stickstoff-Verhältnisses nur sehr schwer. Lärchen bilden daher jährlich große Mengen an Rohhumus und tragen somit zur Versauerung und folglich zur Bodenverschlechterung bei. Dementsprechend stellt sich in forstlichen Lärchen-Monokulturen ein entsprechend bodensaurer Unterwuchs ein. Die Nadelblätter sind leicht giftig, sie enthalten 0,2\% ätherische Öle (DüLL \& KutZelnigg 2011).

\subsection{Zapfen}

Lärchen sind einhäusig, männliche und weibliche Zapfen werden auf der gleichen Pflanze im März gebildet. Die männlichen Blüten (auch Pollenzapfen genannt) sind kugelig bis eiförmig. Sie umfassen zahlreiche, spiralig stehende Staubblätter, die aus einem Stiel, einer blattartigen Struktur und den Pollensäcken bestehen. Die für Kieferngewächse typischen Luftsäcke an den Pollenkörnern fehlen.

Die weiblichen, eiförmigen Zapfen (auch Samenzapfen genannt) werden nach der Freisetzung der Samen nicht abgeworfen, sondern bleiben über Jahre hinweg am Baum. Sie bestehen aus zahlreichen sog. Deck-/Samenschuppen-Komplexen. Die Samenschuppe steht dabei in der Achsel der Deckschuppe (vgl. DöRken \& JaGel 2010). Der Lärchenzapfen entspricht einem verzweigten Blütenstand. Auf

Abb. 6 (oben): Lärchen mit gelber Herbstfärbung der Nadeln.

Abb. 7 (unten): Kahle Lärche im Winter.
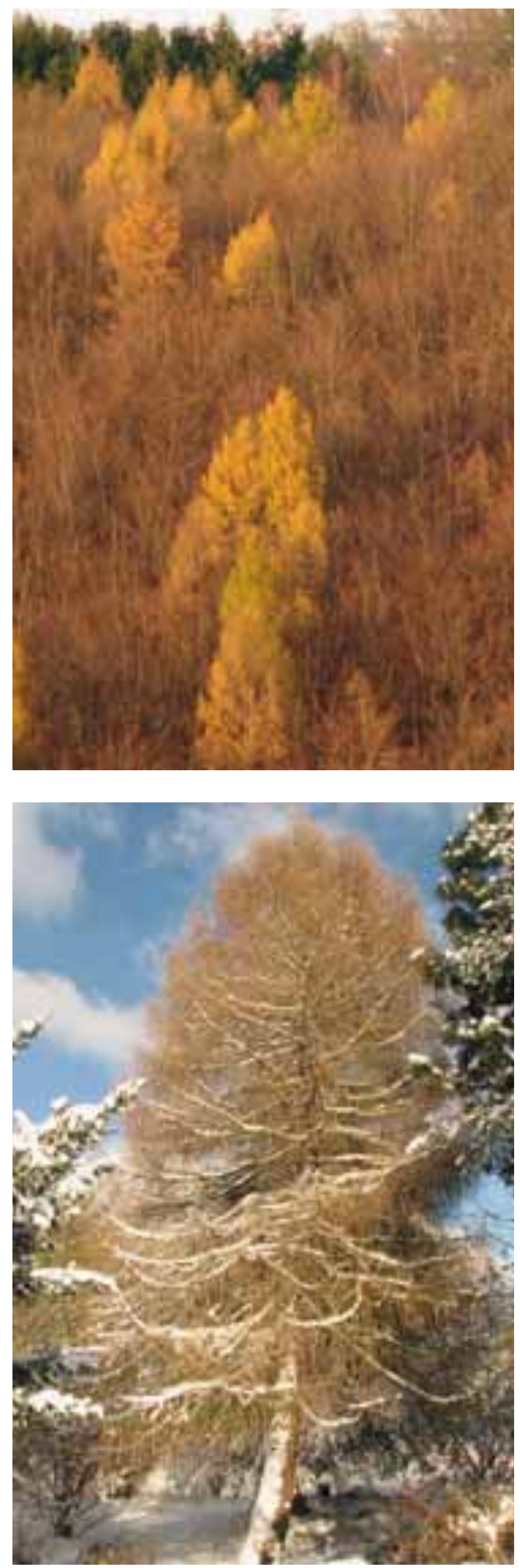

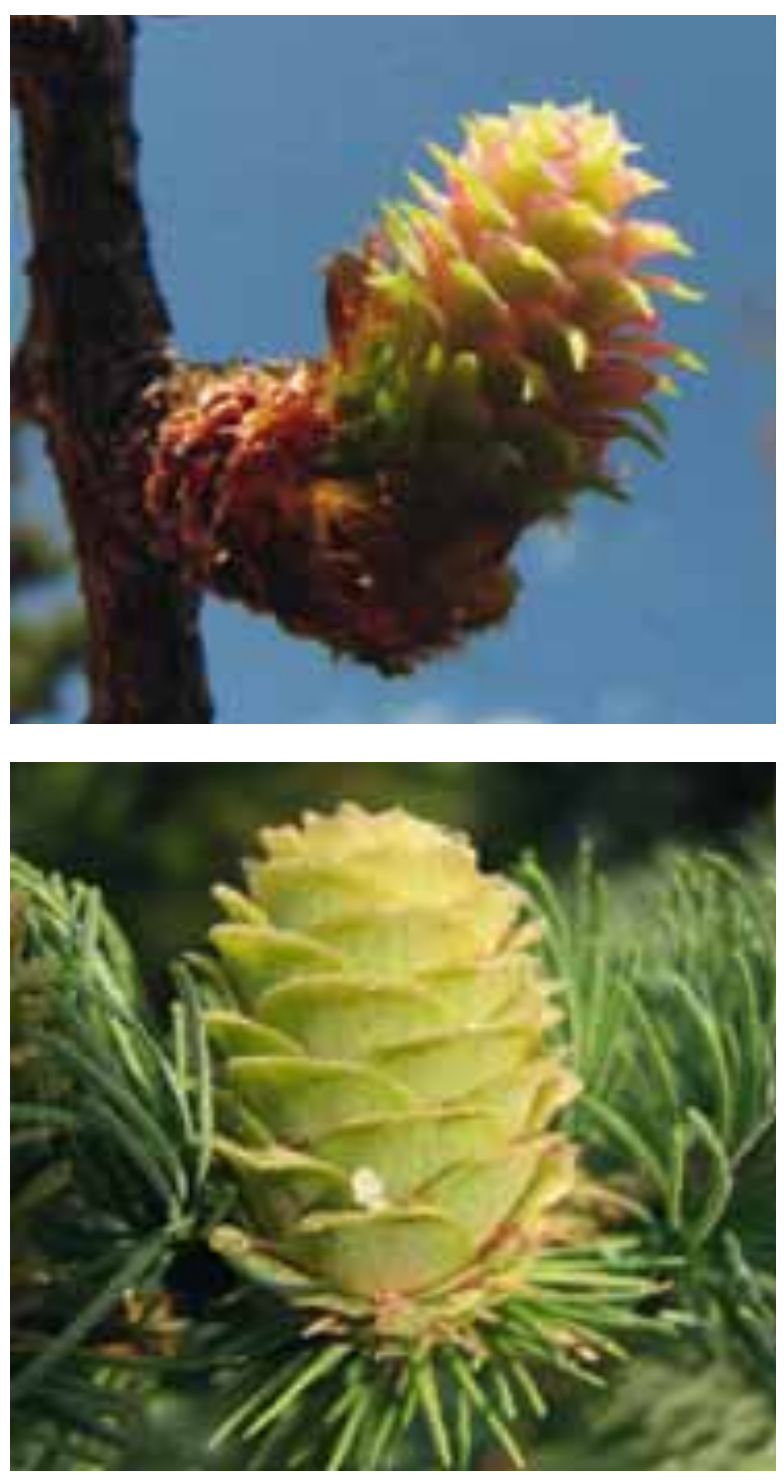

jeder Samenschuppe stehen zwei Samenanlagen. Lärchen sind wie alle Koniferen windbestäubt. Einen Bestäubungstropfen, wie er bei den meisten anderen Koniferen ausgebildet wird (vgl. JAGEL 2011), gibt es bei Lärchen aber nicht. Die Samenschuppe ist zum Zeitpunkt der Blüte deutlich kürzer als die Deckschuppe. Nach der Bestäubung wächst sie stark heran und überragt zum Zeitpunkt der Samenreife die Deckschuppe um ein Vielfaches. Letztere ist im reifen Zapfen von außen nicht mehr erkennbar. Die Samenreife erfolgt im Jahr der Bestäubung im Oktober. Reife Lärchensamen bekommen von der Samenschuppe Gewebe aufgelagert, wodurch ein Samenflügel entsteht. Die Samen werden im späten Herbst vom Wind ausgebreitet.

\section{Verwendung}

Lärchenholz ist ein wichtiger Rohstoff. Das Holz der Europäischen Lärche gehört mit zu den härtesten und beständigsten Nadelhölzern der mitteleuropäischen Flora. Es ist sogar unter Wasser recht beständig. Lärchenholz wird besonders häufig für Treppen, Türen und Fenster genutzt, aber auch als Wand- und Deckenverkleidung (Sснüтt et al. 2002). Daher wird die Lärche häufig angebaut und ist heute auch in Tieflandregionen verbreitet. Lärchenforste sind im Gegensatz zu Fichtenforsten wesentlich lichtdurchfluteter, sodass auch ein Unterwuchs und eine Entwicklung einer ausgeprägten Krautschicht möglich sind. Die Lärche wird z. B. auf Pionierstandorten (wie Lawinenzügen) zur Erstbegrünung eingesetzt. In Parkanlagen und großen Gärten wird sie aufgrund des auffällig hellgrünen Austriebs und der leuchtend gelben Herbstfärbung oft als Solitärbaum gepflanzt. Lärchen sind sehr harzreiche Bäume, deren Harze bereits im Altertum kommerziell gewonnen und aufgrund des hohen Terpentingehaltes (10-25\%) unter der Bezeichnung Venezianisches Terpentin geführt wurden (DüLl \& Kutzelnigg 2011). Neben der heimischen Lärche, Larix decidua var. decidua, wird auch die Japanische Lärche (Larix kaempferi) sowie die Hybride der beiden Arten die Hybrid-Lärche (Larix $\mathrm{x}$ eurolepis) als Forstbaum in M-Europa angepflanzt.

\section{Weitere in Mitteleuropa angebaute Lärchen-Arten}

\subsection{Larix kaempferi - Japanische Lärche}

Die Japanische Lärche ist in Japan auf niederschlagsreichen, vulkanischen Berghängen und in kalten Gebirgslagen zwischen 1100 bis $1900 \mathrm{~m}$ ü.NN heimisch. Sie wird in Mitteleuropa besonders aufgrund ihrer Anspruchslosigkeit und ihrer Resistenz gegenüber dem gefürchteten Lärchenkrebs (Lachnellula willkommii) forstwirtschaftlich angebaut. Im Gegensatz zur Europäischen Lärche ist die Japani-

Abb. 8 (oben): Junger Zapfen von Larix kaempferi.

Abb. 9 (unten): Älterer Zapfen von Larix kaempferi. 
sche Lärche aber etwas gefährdeter gegenüber Schneebruch und reagiert noch empfindlicher auf Trockenstress. Zur Unterscheidung von $\mathrm{La}$ rix decidua zieht man im Wesentlichen die weniger auffällig roten Zapfen, die rötliche Farbe der jungen Zweige sowie Länge und Farbe der Nadeln (blaugrün und wachsbereift) sowie die Zapfenform heran.

\subsection{Larix $\mathrm{x}$ eurolepis (L. decidua $\mathrm{x}$ L. kaempferi) - Hybrid-Lärche}

Die Hybrid-Lärche ist eine Kreuzung aus der Europäischen (L. decidua) und der Japanischen (L. kaempferi) Lärche, die um 1900 im Park Dunkeld (Pirthshire, Schottland) entstand (Schütт et al. 2002). In ihr sind die bessere Anpassung an Winter (geringere Schneebruchgefährdung) von $L$. decidua kombiniert mit der Lärchenkrebs-Resistenz von L. kaempferi, weswegen sie auch bei uns ein wertvoller Forstbaum ist. Bezüglich ihrer Merkmale steht die Hybride zwischen den Eltern und ist oft nur sehr schwer von ihnen zu unterscheiden. Für optimale Wuchsergebnisse, bedingt durch den Heterosiseffekt (Hybriden sind starkwüchsiger als die Elternarten) ist in der Forstwirtschaft die Verwendung von F1-Hybriden (Hybriden der ersten Tochtergeneration) unerlässlich, denn nur bei diesen schlägt dieser positive Bastardierungseffekt in seiner vollen Ausprägung durch.

\section{Literatur}

DöRKEN, V. M. 2012: The evolutionary relevance of vegetative long-shoot/short-shoot differentiation in gymnospermous tree species. - Bibliotheca Botanica Bib. Bot. 161. - Stuttgart.

Dörken, V. M. \& Jagel, A. 2010: Weihnachtliche Koniferenzapfen. - Jahrb. Bochumer Bot. Ver. 1: 270-281. Düll, R. \& Kutzelnigg, H. 2011: Taschenlexikon der Pflanzen Deutschlands und angrenzender Länder. Wiebelsheim.

JAGEL, A. 2011: Die Koniferen blühen - Bei den Zypressengewächsen eine glitzernde Mikrowelt. Palmengarten 75: 137-144.

Abb. 10 (oben): Ausgereifter Zapfen von Larix kaempferi. Abb. 11 (unten): Reifer Zapfen von Larix x eurolepis. 